\title{
FACTORES SOCIOECONÓMICOS QUE SE RELACIONAN CON LA TASA DE DIVORCIOS DE LA MUJER EN EL PERÚ
}

\author{
SOCIOECONOMIC FACTORS RELATED TO THE DIVORCE RATE OF WOMAN IN PERU
}

\section{RESUMEN}

Objetivo: Determinar la relación que existe entre la tasa de divorcio de la mujer y sus diferentes factores socioeconómicos en los departamentos del Perú. Método: La investigación fue de tipo cuantitativo no experimental y transversal; de alcance descriptivo, correlacional y explicativo; como población se usaron los datos macroeconómicos de las mujeres de los 24 departamentos del Perú, información obtenida de las series estadísticas de XII Censo de Población, VII de Vivienda y III de Comunidades Indígenas o Censo peruano de 2017. Resultados: La tasa de divorcio y la fuerza laboral femenina con educación superior tiene una correlación positiva fuerte de $74.80 \%$. La tasa de divorcio y jefa de hogar con alto poder adquisitivo presentaron una correlación directa y significativa de $96.8 \%$. La tasa de divorcio y urbanidad presentaron una correlación directa y positiva de $64.70 \%$. Conclusión: La tasa de divorcio presenta una alta correlación directa y significativa con la fuerza laboral femenina, con el poder adquisitivo y la urbanidad.

Palabras clave: Tasa de divorcio; fuerza laboral femenina; educación superior; poder adquisitivo y urbanidad.
Antony Raúl Stuardo Castillo Chiroque Universidad Privada Antenor Orrego Trujillo, Perú

ORCID: https://orcid.org/0000-0002-4857-4543

Correo electrónico: acastilloc9@upao.edu.pe

Ena Cecilia Obando Peralta Universidad Privada Antenor Orrego Trujillo, Perú

ORCID: https://orcid.org/0000-0001-5734-6764 Correo electrónico: eobandop@upao.edu.pe

Edmundo Rafael Casavilca Maldonado Universidad Nacional Mayor de San Marcos Lima, Perú

ORCID: https://orcid.org/0000-0001-8625-9811

Correo electrónico: ecasavilcam@gmail.com

[Recibido: 25/04/2021 Aceptado: 02/07/2021 Publicado: 31/08/2021]

\begin{abstract}
Objective: To determine the relationship between socioeconomic factors and the divorce rate of women in the departments of Peru. Method: The research was quantitative, non-experimental, and cross-sectional; descriptive, correlational, and explanatory in scope. The population was seized from the macroeconomic data of the women of Peru's 24 departments, data that was obtained from the statistical series of the XII Population Census, VII Housing and III Indigenous Communities or Peruvian Census of 2017. Results: Divorce rate and the female workforce with higher education have a strong positive correlation of $74.80 \%$; Divorce rate and head of household with high purchasing power presented a direct and significant correlation of $96.8 \%$; Divorce rate and urbanity presented a direct and positive correlation of $64.70 \%$. Conclusion: The divorce rate has a high direct and significant correlation with the female labor force, with purchasing power, and urbanity.
\end{abstract}

Keywords: Divorce rate; female workforce; higher education; purchasing power and civility. 


\section{INTRODUCCIÓN}

El presente estudio pretende determinar la relación que existe entre la tasa de divorcio de la mujer y sus diferentes factores socioeconómicos en los departamentos del Perú. Se considera como factores socioeconómicos la fuerza laboral femenina, el poder adquisitivo y la urbanidad. Por ello, se planteó como hipótesis que la tasa de divorcio presenta una alta correlación directa y significativa con la fuerza laboral femenina, con el poder adquisitivo y la urbanidad.

El matrimonio es un suceso muy importante en la vida de un ser humano. Al constituir una familia, se comparten costumbres y se distribuyen roles que corresponden a cada miembro del hogar. A partir del año 2000, el matrimonio empezó a ser menos visto como una unión espiritual sagrada, y comenzó a ser considerado como un compromiso personal y práctico que puede romperse si fracasa. Es claro que no todos los matrimonios tienen éxito, algunos terminan por falta de armonía (Benítez, 2017).

El divorcio tiene un impacto profundo en la vida de los cónyuges, hijos y la sociedad en general. Este también puede crear dificultades financieras, especialmente para las mujeres que dependen de su esposo; pues el divorcio implica para la mujer, en la mayoría de los casos, aceptar nuevas rutinas y obligaciones que no se tenían hasta ese momento, ya que en la mayoría de los casos son ellas quienes se quedan con la custodia de sus hijos. Estos distintos y repentinos cambios repercuten en nuevos roles de acuerdo con la nueva composición familiar (Moine, 2013).

Desde la década de los 50, las mujeres que no tenían estudios completos o nunca habían trabajado eran las más perjudicadas en un divorcio. La mujer era criada para cumplir con los deberes de la casa y, al ser incapaz de generar ingresos para mantener su hogar, era más difícil separarse de su cónyugue (Ailes, 2020).

Según Guzmán, Reyes, Castillo y Cantos (2020), la consolidación de las mujeres en la instrucción formal resumida y el notable incremento de su apoyo en la publicidad del trabajo ha impulsado el logro de la libertad monetaria para un número significativo de ellas, junto con el dominio político, social y familiar, entre otras diferentes perspectivas que sugiere esta circunstancia.

Según De la Piedra (2017), aunque las mujeres pudieron reclamar sus derechos y hacerse valer por sí mismas, el hombre y la sociedad se imponen y menosprecian la capacidad intelectual que ellas tienen. Muchas veces se menciona que las oportunidades laborales son las mismas para el hombre y la mujer; sin embargo, la realidad es otra y así lo precisan los indicadores. Pues las mujeres aún continúan contribuyendo muy poco en el PBI y, en 13 años, el porcentaje de mujeres en un puesto de liderazgo ha incrementado en tan solo un $25 \%$.

En un matrimonio se espera una convivencia igualitaria. El esposo debería ser la principal ayuda para su esposa, no solo en el rol de quehaceres del hogar, sino también en el ámbito laboral; al igual que una ayuda para vencer los diversos tipos de obstáculos que se presentan en las organizaciones, especialmente, si la mujer está en una posición que le permitirá adquirir un avance empresarial.

Es importante que en un matrimonio exista la armonía y tranquilidad para que así la mujer pueda enfrentar los distintos retos profesionales de responsabilidad empresarial. Si no existe tal apoyo, esto podría ser un motivo para que la mujer decida considerar el divorcio.

No hay divorcios iguales, pues cada ruptura es diferente para cada situación familiar. Esta es una de las razones por las cuales existen diferentes estudios relacionados con el tema; asimismo, los divorcios pueden tener diferentes efectos socioeconómicos en la familia y esta, como célula integrante, puede generar efectos socioeconómicos en la sociedad.

Uchida, Araki y Murata (2019) analizaron los efectos de los bajos ingresos sobre las tasas de matrimonios y divorcio de la población japonesa en 46 prefecturas entre los años 1970 y 1975. Según los estudios, durante este período, la sociedad japonesa experimentó un drástico cambio, de un crecimiento económico duradero a una grave recesión en 1973. En tanto, en 1970 como en 1975, la primera tasa de matrimonios para mujeres estaba inversamente relacionada con los bajos ingresos y las tasas de divorcio para hombres y mujeres fueron relacionadas con bajos ingresos. La tasa de matrimonio y de divorcio para las mujeres aumentaron significativamente; estos hallazgos sugieren que un bajo ingreso es el factor esencial que afecta el matrimonio y repercute en el divorcio para las mujeres.

De acuerdo con Suárez (2017), el problema que han tenido las mujeres dentro del matrimonio era la falta de igualdad, pues se otorgaba un estatus superior al hombre; sin embargo, a lo largo de la historia ello ha ido cambiando, logrando que la mujer tenga un rol más significativo en los círculos sociales, monetarios y políticos.

Por último, Tamez y Ribeiro (2016) presentan una investigación sobre la separación como un fenómeno familiar y social, fijada en los primordiales impactos estudiados y su efecto diferencial en las personas. Para el estudio, se utilizó la información primaria de una investigación de intento de separación en Nuevo León. Las cualidades de esta información actual, según la hipótesis del cambio estadístico, comprenden un marcador del cambio familiar y social visto en las décadas en curso. 
Esto permite una apreciación económica del vínculo que existe entre la familia y la economía, ya que es evidente que cada individuo de la familia asume un trabajo fundamental en la economía y en la familia, y cada una de sus manifestaciones puede tener un efecto positivo o negativo en la economía doméstica (Suárez, 2017).

Las mujeres tienen un trabajo participativo cada vez más importante en los aspectos socioeconómicos, demostrando que pasaron de una economía de subsistencia donde invirtieron toda su energía en el hogar, a una economía generadora de salarios, en la cual consiguen el trabajo en el mercado de manera más efectiva y con la presentación de empleos administrativos progresivos, dándose cambios en la familia y en la economía.

\section{MATERIAL Y MÉTODOS}

La población estuvo compuesta por la serie anual de los 24 departamentos del Perú, la cual fue obtenida de las series estadísticas de los Censos Nacionales 2017: XII de Población, VII de Vivienda y III de Comunidades Indígenas (Instituto Nacional de Estadística e Informática [INEI], 2018).

La investigación fue de carácter explicativo-correlacional, transversal y no experimental. Se utilizaron como técnicas el análisis documental y búsqueda en Internet, y como instrumentos las fichas bibliográficas, hojas de registro y archivos Excel. Para comprobar la hipótesis planteada, se aplicó una regresión lineal a través de la técnica de los Mínimos Cuadrado Ordinarios.

\section{RESULTADOS}

El empleo que consiguen las mujeres depende mucho de su grado de educación; es así que el Censo Nacional realizado por el INEI el 2017 contempla determinar la PEA femenina, teniendo en cuenta los diferentes grados de educación de las mujeres, los cuales son: sin nivel educativo, con educación primaria, con educación secundaria y con educación superior. Para el presente trabajo de investigación se considera que, a mayor nivel de ingresos, la mujer es más propensa a divorciarse, en el sentido de que no tendrá la necesidad de depender de su esposo económicamente.

Según el Censo Nacional del INEI (2018), la PEA total fue de 12 millones 305 mil 718 habitantes y la PEA clasificada entre hombres y mujeres fue: la PEA masculina 7 millones 539 mil 062 y la PEA femenina 4 millones 766 mil 656.

Los departamentos que mayor empleabilidad mostraron respecto a las mujeres con educación superior fueron: Lima con un 53.86\% ( 1 millón 136 mil 838 mujeres), Arequipa con un 52.98\% (146 mil 645 mujeres) y, finalmente, el departamento de Moquegua con un 52.62\% (17 mil 708 mujeres), los cuales se encuentran entre los tres primeros departamentos que concentran la mayor fuerza laboral femenina. La oferta laboral femenina con educación superior que presentan estos departamentos significa una oportunidad para cambiar sus vidas y desarrollar su potencial como un factor de progreso en el ámbito social, político y económico; buscando entidades u organizaciones en las que no solo contribuyan al mejor desempeño de sus capacidades y habilidades en el mercado laboral, sino también una cultura progresiva, en la que los valores como la honestidad y la ética ganen tanto en el interior como en el exterior.

Los departamentos que menor empleabilidad mostraron respecto a las mujeres con educación superior fueron: Loreto con un 32.24\% (35 mil 623 mujeres), Huancavelica con un 31.40\% (12 mil 275 mujeres) y, por último, el departamento de Puno con un 30.66\% (55 mil 440 mujeres), quienes se encuentran entre los tres últimos departamentos que concentran la menor fuerza laboral femenina. Estos departamentos tienen por lo menos un tercio de su población en situación de pobreza, lo que refleja la relación de la fuerza laboral femenina con un bajo nivel de educación secundaria y superior. Asimismo, estas mujeres que se encuentran bajo estas condiciones se dedican mayormente a actividades orientadas al sector agropecuario, el cual se caracteriza por ser poco productivo para el desarrollo social y económico de estos departamentos; mientras que una cantidad menor de mujeres se dedica al sector comercio que aporta de manera escasa a la economía. Lo descripto se puede evidenciar en la Tabla 1.

Los departamentos con mayor porcentaje de mujeres como jefas de hogar con alto poder adquisitivo fueron: Lima con un 11.58\% (122 mil 223 mujeres), Arequipa con un $9.22 \%$ (15 mil 280 mujeres) y, finalmente, el departamento de Moquegua con un 6.52\% (1 mil 452 mujeres), los cuales se encuentran entre los tres primeros departamentos que concentran un alto poder adquisitivo. En estos departamentos, la educación básica, secundaria y superior, ha traído mayor influencia, habilidades, confianza en sí mismas y expectativas. Hecho que aporta a su rol como jefas de hogar un alto poder adquisitivo, pues son mujeres que obtuvieron títulos universitarios y experiencia laboral las que están preparadas para transformar el lugar de trabajo y, a su vez, el mercado.

Asimismo, los departamentos que tienen menor porcentaje de jefas de hogar con alto poder adquisitivo fueron: Puno con un 1.05\% (1 mil 665 mujeres), Amazonas con un $0.77 \%$ (225 mujeres) y, finalmente, el departamento de Huancavelica con un $0.29 \%$ (107 mujeres). Estos se encuentran entre los tres últimos departamentos que no concentran un alto poder adquisitivo, ya que en estos departamentos las mujeres no encuentran un mercado 
Tabla 1

Distribución del mercado laboral según sexo y niveles educativos (Porcentaje)

\begin{tabular}{|c|c|c|c|c|c|c|c|}
\hline \multirow[b]{3}{*}{$\mathbf{N}^{\circ}$} & \multirow[b]{3}{*}{ Departamentos } & \multirow{3}{*}{$\begin{array}{c}\text { PEA } \\
\text { Hombre }\end{array}$} & \multicolumn{5}{|c|}{ PEA } \\
\hline & & & \multicolumn{5}{|c|}{ Mujeres } \\
\hline & & & Sin Nivel & Educación Básica ${ }^{1 /}$ & $\begin{array}{l}\text { Educación } \\
\text { Secundaria }\end{array}$ & $\begin{array}{l}\text { Educación } \\
\text { Superior }{ }^{2 /}\end{array}$ & Total \\
\hline 1 & Departamento de Amazonas & $71.04 \%$ & $7.25 \%$ & $32.67 \%$ & $24.04 \%$ & $36.04 \%$ & $28.96 \%$ \\
\hline 2 & Departamento de Ancash & $65.67 \%$ & $4.77 \%$ & $17.44 \%$ & $31.74 \%$ & $46.06 \%$ & $34.33 \%$ \\
\hline 3 & Departamento de Apurímac & $65.50 \%$ & $11.32 \%$ & $19.76 \%$ & $27.42 \%$ & $41.50 \%$ & $34.50 \%$ \\
\hline 4 & Departamento de Arequipa & $57.09 \%$ & $2.22 \%$ & $13.21 \%$ & $31.59 \%$ & $52.98 \%$ & $42.91 \%$ \\
\hline 5 & Departamento de Ayacucho & $62.70 \%$ & $10.40 \%$ & $23.11 \%$ & $28.76 \%$ & $37.73 \%$ & $37.30 \%$ \\
\hline 6 & Departamento de Cajamarca & $71.01 \%$ & $10.59 \%$ & $29.94 \%$ & $22.20 \%$ & $37.27 \%$ & $28.99 \%$ \\
\hline 7 & Departamento de Cusco & $60.03 \%$ & $8.38 \%$ & $19.76 \%$ & $29.31 \%$ & $42.55 \%$ & $39.97 \%$ \\
\hline 8 & Departamento de Huancavelica & $65.09 \%$ & $16.01 \%$ & $28.80 \%$ & $23.79 \%$ & $31.40 \%$ & $34.91 \%$ \\
\hline 9 & Departamento de Huánuco & $68.11 \%$ & $8.28 \%$ & $23.11 \%$ & $27.02 \%$ & $41.59 \%$ & $31.89 \%$ \\
\hline 10 & Departamento de Ica & $60.21 \%$ & $1.11 \%$ & $10.89 \%$ & $37.24 \%$ & $50.76 \%$ & $39.79 \%$ \\
\hline 11 & Departamento de Junín & $60.62 \%$ & $4.73 \%$ & $20.46 \%$ & $32.04 \%$ & $42.77 \%$ & $39.38 \%$ \\
\hline 12 & Departamento de La Libertad & $64.42 \%$ & $3.14 \%$ & $18.48 \%$ & $30.66 \%$ & $47.72 \%$ & $35.58 \%$ \\
\hline 13 & Departamento de Lambayeque & $64.08 \%$ & $2.77 \%$ & $16.55 \%$ & $32.69 \%$ & $47.99 \%$ & $35.92 \%$ \\
\hline 14 & Departamento de Lima & $57.63 \%$ & $0.90 \%$ & $9.27 \%$ & $35.97 \%$ & $53.86 \%$ & $42.37 \%$ \\
\hline 15 & Departamento de Loreto & $63.25 \%$ & $4.83 \%$ & $27.77 \%$ & $35.16 \%$ & $32.24 \%$ & $36.75 \%$ \\
\hline 16 & Departamento de Madre de Dios & $64.20 \%$ & $2.63 \%$ & $18.28 \%$ & $39.55 \%$ & $39.54 \%$ & $35.80 \%$ \\
\hline 17 & Departamento de Moquegua & $59.16 \%$ & $2.83 \%$ & $14.67 \%$ & $29.87 \%$ & $52.62 \%$ & $40.84 \%$ \\
\hline 18 & Departamento de Pasco & $67.79 \%$ & $5.79 \%$ & $22.06 \%$ & $29.51 \%$ & $42.64 \%$ & $32.21 \%$ \\
\hline 19 & Departamento de Piura & $68.23 \%$ & $2.95 \%$ & $18.40 \%$ & $30.45 \%$ & $48.19 \%$ & $31.77 \%$ \\
\hline 20 & Departamento de Puno & $60.25 \%$ & $9.08 \%$ & $28.17 \%$ & $32.09 \%$ & $30.66 \%$ & $39.75 \%$ \\
\hline 21 & Departamento de San Martin & $68.98 \%$ & $5.42 \%$ & $30.18 \%$ & $28.97 \%$ & $35.44 \%$ & $31.02 \%$ \\
\hline 22 & Departamento de Tacna & $56.02 \%$ & $2.34 \%$ & $16.62 \%$ & $36.74 \%$ & $44.30 \%$ & $43.98 \%$ \\
\hline 23 & Departamento de Tumbes & $66.67 \%$ & $1.14 \%$ & $15.41 \%$ & $35.27 \%$ & $48.19 \%$ & $33.33 \%$ \\
\hline 24 & Departamento de Ucayali & $64.05 \%$ & $3.69 \%$ & $21.51 \%$ & $38.27 \%$ & $36.53 \%$ & $35.95 \%$ \\
\hline & TOTAL & $61.26 \%$ & $3.30 \%$ & $15.41 \%$ & $33.27 \%$ & $48.02 \%$ & $38.74 \%$ \\
\hline
\end{tabular}

${ }^{2 /}$ Comprende No Universitaria, Universitaria y Post Grado

${ }^{1 /}$ Comprende Formación Inicial y Primaria Regular, y Básica Especial

Fuente: Elaboración propia con base en el Instituto Nacional de Estadística e Informática (2018)

laboral bien remunerado o no reciben estudios básicos, secundarios y superiores, haciendo que muchas de estas mujeres a temprana edad, formen su familia y decidan dedicarse a las tareas diarias del hogar, la formación de los hijos y a la atención de su esposo. Lo descripto se puede evidenciar en la Tabla 2.

Las cifras presentadas por el Censo Nacional del INEI (2018) muestran que, en el Perú, la población urbana se encontró en un $79.34 \%$ y la población rural en $20.66 \%$. En la Tabla 3, se muestra los resultados censales de lo rural y urbano en los distintos departamentos del Perú. Los departamentos con mayor porcentaje en urbanidad son: Lima con un $98.47 \%$, Tumbes con un $93.65 \%$, Ica con un $92.44 \%$, Arequipa con un $91.77 \%$ y, por último, el departamento de Tacna con un $90.12 \%$.

Según el Censo Nacional del INEI (2018), la población femenina según su estado civil o conyugal fue de $14 \mathrm{mi}-$ llones 931 mil 127 mujeres; en situación de divorcio fue de 125 mil 39 mujeres, y otros (convivientes, separadas, casadas, viudas y solteras) fue de 14 millones 806 mil 88 . 
Tabla 2

Poder adquisitivo de la jefa de hogar (Porcentaje)

\begin{tabular}{|c|c|c|c|c|}
\hline \multirow[b]{2}{*}{$\mathbf{N}^{\circ}$} & \multirow[b]{2}{*}{ Departamentos } & \multicolumn{3}{|c|}{ Mujeres } \\
\hline & & $\begin{array}{l}\text { Jefas de hogar con alto } \\
\text { poder adquisitivo }\end{array}$ & $\begin{array}{c}\text { Jefas de hogar con bajo y mediano } \\
\text { poder adquisitivo }\end{array}$ & $\begin{array}{l}\text { Jefas de } \\
\text { hogar }\end{array}$ \\
\hline 1 & Departamento de Amazonas & $0.77 \%$ & $99.23 \%$ & $100 \%$ \\
\hline 2 & Departamento de Ancash & $3.02 \%$ & $96.98 \%$ & $100 \%$ \\
\hline 3 & Departamento de Apurímac & $1.39 \%$ & $98.61 \%$ & $100 \%$ \\
\hline 4 & Departamento de Arequipa & $9.22 \%$ & $90.78 \%$ & $100 \%$ \\
\hline 5 & Departamento de Ayacucho & $1.22 \%$ & $98.78 \%$ & $100 \%$ \\
\hline 6 & Departamento de Cajamarca & $1.56 \%$ & $98.44 \%$ & $100 \%$ \\
\hline 7 & Departamento de Cusco & $3.97 \%$ & $96.03 \%$ & $100 \%$ \\
\hline 8 & Departamento de Huancavelica & $0.29 \%$ & $99.71 \%$ & $100 \%$ \\
\hline 9 & Departamento de Huánuco & $1.42 \%$ & $98.58 \%$ & $100 \%$ \\
\hline 10 & Departamento de Ica & $4.11 \%$ & $95.89 \%$ & $100 \%$ \\
\hline 11 & Departamento de Junín & $2.69 \%$ & $97.31 \%$ & $100 \%$ \\
\hline 12 & Departamento de La Libertad & $4.94 \%$ & $95.06 \%$ & $100 \%$ \\
\hline 13 & Departamento de Lambayeque & $3.58 \%$ & $96.42 \%$ & $100 \%$ \\
\hline 14 & Departamento de Lima & $11.58 \%$ & $88.42 \%$ & $100 \%$ \\
\hline 15 & Departamento de Loreto & $1.12 \%$ & $98.88 \%$ & $100 \%$ \\
\hline 16 & Departamento de Madre de Dios & $2.09 \%$ & $97.91 \%$ & $100 \%$ \\
\hline 17 & Departamento de Moquegua & $6.52 \%$ & $93.48 \%$ & $100 \%$ \\
\hline 18 & Departamento de Pasco & $1.33 \%$ & $98.67 \%$ & $100 \%$ \\
\hline 19 & Departamento de Piura & $2.82 \%$ & $97.18 \%$ & $100 \%$ \\
\hline 20 & Departamento de Puno & $1.05 \%$ & $98.95 \%$ & $100 \%$ \\
\hline 21 & Departamento de San Martin & $1.52 \%$ & $98.48 \%$ & $100 \%$ \\
\hline 22 & Departamento de Tacna & $6.17 \%$ & $93.83 \%$ & $100 \%$ \\
\hline 23 & Departamento de Tumbes & $2.61 \%$ & $97.39 \%$ & $100 \%$ \\
\hline \multirow[t]{2}{*}{24} & Departamento de Ucayali & $1.57 \%$ & $98.43 \%$ & $100 \%$ \\
\hline & TOTAL & $6.34 \%$ & $93.66 \%$ & $100 \%$ \\
\hline
\end{tabular}

Fuente: Elaboración propia con base en el Instituto Nacional de Estadística e Informática (2018)

Tabla 3

Urbanidad por departamento (Porcentaje)

\begin{tabular}{clcc}
\hline $\mathbf{N}^{\mathbf{0}}$ & Departamentos & Rural & Urbano \\
\hline 1 & Dep. de Amazonas & 58.47 & 41.53 \\
2 & Dep. de Ancash & 36.62 & 63.38 \\
3 & Dep. de Apurímac & 54.17 & 45.83 \\
4 & Dep. de Arequipa & 8.23 & 91.77 \\
5 & Dep. de Ayacucho & 41.89 & 58.11 \\
6 & Dep. de Cajamarca & 64.57 & 35.43 \\
7 & Dep. de Cusco & 39.34 & 60.66 \\
8 & Dep. de Huancavelica & 69.55 & 30.45 \\
9 & Dep. de Huánuco & 47.93 & 52.07 \\
10 & Dep. de Ica & 7.56 & 92.44 \\
\hline
\end{tabular}


Tabla 3. Continuación

\begin{tabular}{llcc}
\hline $\mathbf{N}^{\circ}$ & Departamentos & Rural & Urbano \\
\hline 11 & Dep. de Junín & 28.98 & 71.02 \\
12 & Dep. de La Libertad & 21.06 & 78.94 \\
13 & Dep. de Lambayeque & 18.89 & 81.11 \\
14 & Dep. de Lima & 1.53 & 98.47 \\
15 & Dep. de Loreto & 31.33 & 68.67 \\
16 & Dep. de Madre de Dios & 17.24 & 82.76 \\
17 & Dep. de Moquegua & 13.14 & 86.86 \\
18 & Dep. de Pasco & 36.92 & 63.08 \\
19 & Dep. de Piura & 20.73 & 79.27 \\
20 & Dep. de Puno & 46.22 & 53.78 \\
21 & Dep. de San Martin & 31.88 & 68.12 \\
22 & Dep. de Tacna & 9.88 & 90.12 \\
23 & Dep. de Tumbes & 6.35 & 93.65 \\
24 & Dep. de Ucayali & 19.00 & 81.00 \\
\hline & $\quad$ TOTAL & 20.66 & 79.34 \\
\hline
\end{tabular}

Fuente: Elaboración propia con base en el Instituto Nacional de Estadística e Informática (2018)

Las mujeres en situación de divorcio han aumentado en los distintos departamentos del Perú. El departamento de La Libertad se ubica en la sexta posición con $0.67 \%$ (6 mil 73 mujeres), detrás de Ica con $0.74 \%$ (3 mil 264 mujeres), Tacna con un $0.99 \%$ (mil 639 mujeres), Arequipa con $1.07 \%$ (7mil 842 mujeres), Moquegua con 1.08\% (935 mujeres) y la capital del Perú, Lima con 1.35\% (72 mil 430 mujeres). Lo descripto se puede mostrar en la Tabla 4.

La relación entre el empleo de las mujeres y el riesgo de divorcio es compleja y controversial. Cabe resaltar que, la oportunidad de trabajo para las mujeres no debilita intrínsecamente el matrimonio, sino que les proporciona recursos que pueden utilizar para dejar matrimonios insatisfactorios.

Según los datos del Censo Nacional del INEI (2018), en la Figura 1 se muestra una dispersión donde se aprecia una correlación positiva fuerte $(74.80 \%)$ entre la fuerza laboral femenina con educación superior y la tasa de divorcio; es decir, a medida que la fuerza laboral femenina con educación superior aumenta, también lo hace la tasa de divorcio. De igual manera, si hay menos fuerza laboral femenina, la tasa de divorcio disminuye.

Las tasas de divorcio en el Perú han estado aumentando durante la última década, al igual que las tasas de participación femenina en la fuerza laboral. La incorporación de la mujer en el mercado laboral hace que obtenga mayor poder adquisitivo; por lo cual, genera que la tasa de divorcio incremente, siendo en las zonas urbanas donde se concentra más este aumento del divorcio, ya que la mujer considera que no debería someterse a las reglas del hombre. Por ello, para la mujer, independizarse es la mejor opción, como también buscar nuevos ambientes o, con el tiempo, tener otra pareja con la finalidad de tener un bienestar propio.)

Según la información del Censo Nacional del INEI (2018), en la Figura 2 se muestra una dispersión donde se aprecia que las tasas de divorcio están correlacionadas con las tasas de las jefas de hogar con alto poder adquisitivo, siendo dicha relación positiva en $96.8 \%$ (mientras mayor es el poder adquisitivo de la jefa de hogar mayor es la tasa de divorcio). De igual manera, si hay menos poder adquisitivo en la jefa de hogar, la tasa de divorcio disminuye.

En el Perú, las tasas de divorcio son más altas en las ciudades que en las áreas rurales. Si bien se han propuesto muchas explicaciones para esto, una común ha sido la oportunidad económica. Los habitantes de las ciudades generalmente tienen ingresos más altos que sus contrapartes en el país y, tal vez, puedan solventar más fácilmente sus necesidades económicas por sí mismas después de un divorcio. Los habitantes de las ciudades pueden ser más propensos a divorciarse, no porque ganen más dinero, sino porque tienen más y mejores alternativas a su relación actual disponibles en todo momento.

Según los datos del Censo Nacional del INEI (2018), en la Figura 3 se muestra una dispersión donde se aprecia una correlación positiva $(64.70 \%)$ entre urbanidad y la tasa de 
Tabla 4

Población según su estado civil o conyugal (Porcentaje)

\begin{tabular}{|c|c|c|c|c|}
\hline \multirow{2}{*}{$\mathbf{N}^{\circ}$} & \multirow{2}{*}{ Departamentos } & \multicolumn{3}{|c|}{ Mujeres } \\
\hline & & En situación de divorcio & Otros $^{1 /}$ & Población total \\
\hline 1 & Departamento de Amazonas & $0.33 \%$ & $99.67 \%$ & $100.0 \%$ \\
\hline 2 & Departamento de Ancash & $0.54 \%$ & $99.46 \%$ & $100.0 \%$ \\
\hline 3 & Departamento de Apurímac & $0.40 \%$ & $99.60 \%$ & $100.0 \%$ \\
\hline 4 & Departamento de Arequipa & $1.07 \%$ & $98.93 \%$ & $100.0 \%$ \\
\hline 5 & Departamento de Ayacucho & $0.42 \%$ & $99.58 \%$ & $100.0 \%$ \\
\hline 6 & Departamento de Cajamarca & $0.42 \%$ & $99.58 \%$ & $100.0 \%$ \\
\hline 7 & Departamento de Cusco & $0.62 \%$ & $99.38 \%$ & $100.0 \%$ \\
\hline 8 & Departamento de Huancavelica & $0.39 \%$ & $99.61 \%$ & $100.0 \%$ \\
\hline 9 & Departamento de Huánuco & $0.44 \%$ & $99.56 \%$ & $100.0 \%$ \\
\hline 10 & Departamento de Ica & $0.74 \%$ & $99.26 \%$ & $100.0 \%$ \\
\hline 11 & Departamento de Junín & $0.51 \%$ & $99.49 \%$ & $100.0 \%$ \\
\hline 12 & Departamento de La Libertad & $0.67 \%$ & $99.33 \%$ & $100.0 \%$ \\
\hline 13 & Departamento de Lambayeque & $0.62 \%$ & $99.38 \%$ & $100.0 \%$ \\
\hline 14 & Departamento de Lima & $1.35 \%$ & $98.65 \%$ & $100.0 \%$ \\
\hline 15 & Departamento de Loreto & $0.34 \%$ & $99.66 \%$ & $100.0 \%$ \\
\hline 16 & Departamento de Madre de Dios & $0.46 \%$ & $99.54 \%$ & $100.0 \%$ \\
\hline 17 & Departamento de Moquegua & $1.08 \%$ & $98.92 \%$ & $100.0 \%$ \\
\hline 18 & Departamento de Pasco & $0.40 \%$ & $99.60 \%$ & $100.0 \%$ \\
\hline 19 & Departamento de Piura & $0.40 \%$ & $99.60 \%$ & $100.0 \%$ \\
\hline 20 & Departamento de Puno & $0.39 \%$ & $99.61 \%$ & $100.0 \%$ \\
\hline 21 & Departamento de San Martin & $0.42 \%$ & $99.58 \%$ & $100.0 \%$ \\
\hline 22 & Departamento de Tacna & $0.99 \%$ & $99.01 \%$ & $100.0 \%$ \\
\hline 23 & Departamento de Tumbes & $0.46 \%$ & $99.54 \%$ & $100.0 \%$ \\
\hline \multirow[t]{2}{*}{24} & Departamento de Ucayali & $0.39 \%$ & $99.61 \%$ & $100.0 \%$ \\
\hline & TOTAL & $0.84 \%$ & $99.16 \%$ & $100.0 \%$ \\
\hline
\end{tabular}

Nota: ${ }^{1 / C}$ Comprenden convivientes, separadas, casadas, viudas y solteras

Fuente: Elaboración propia con base en el Instituto Nacional de Estadística e Informática (2018)

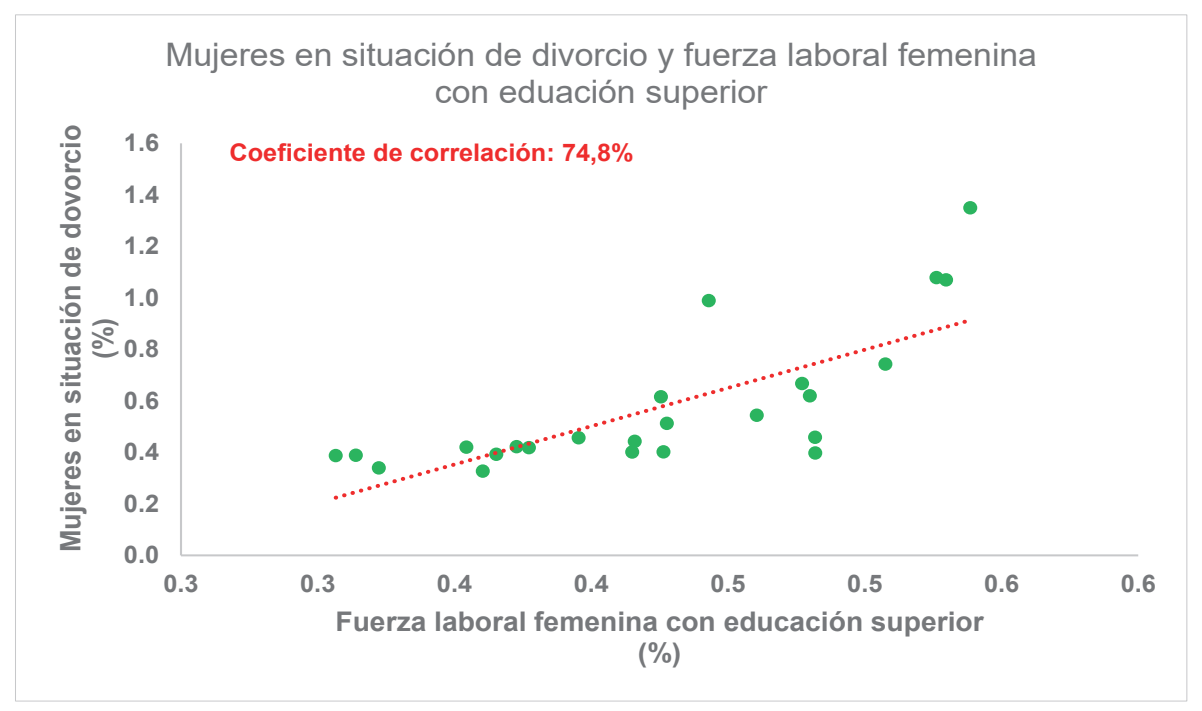

Figura 1. Tasa de divorcio y la fuerza laboral femenina con educación superior

Fuente: Elaboración propia con base en el Instituto Nacional de Estadística e Informática (2018) 


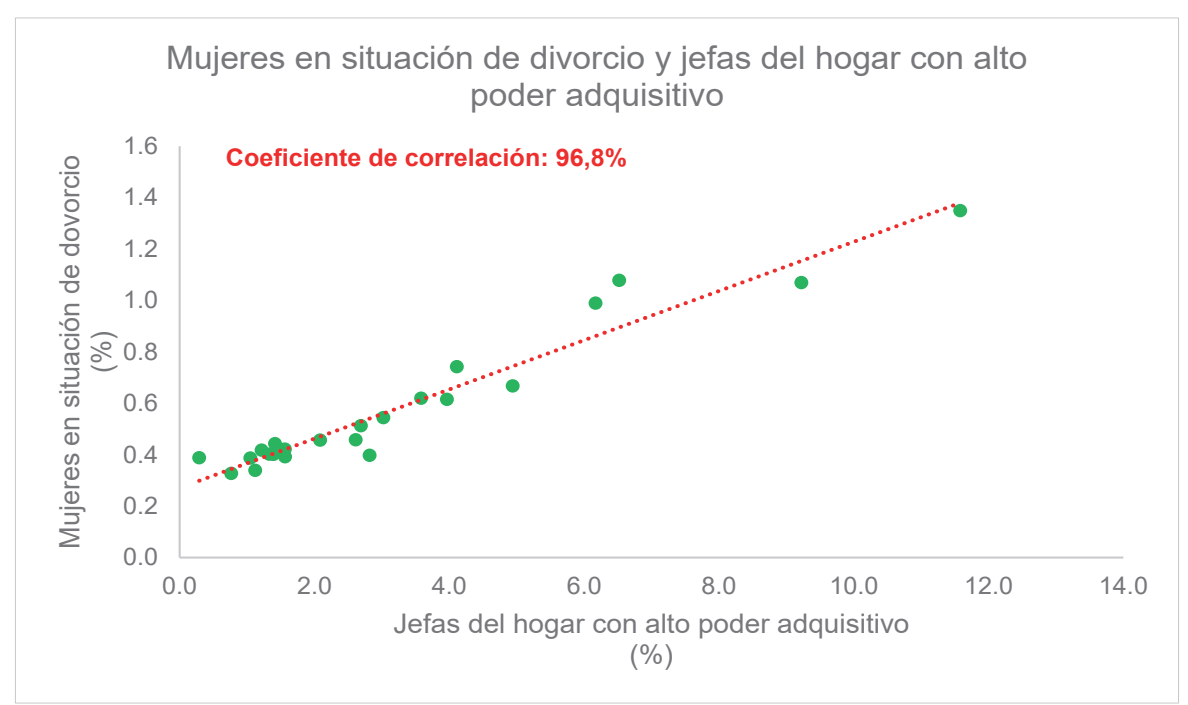

Figura 2. Tasa de divorcio y jefa de hogar con alto poder adquisitivo

Fuente: Elaboración propia con base en el Instituto Nacional de Estadística e Informática (2018)

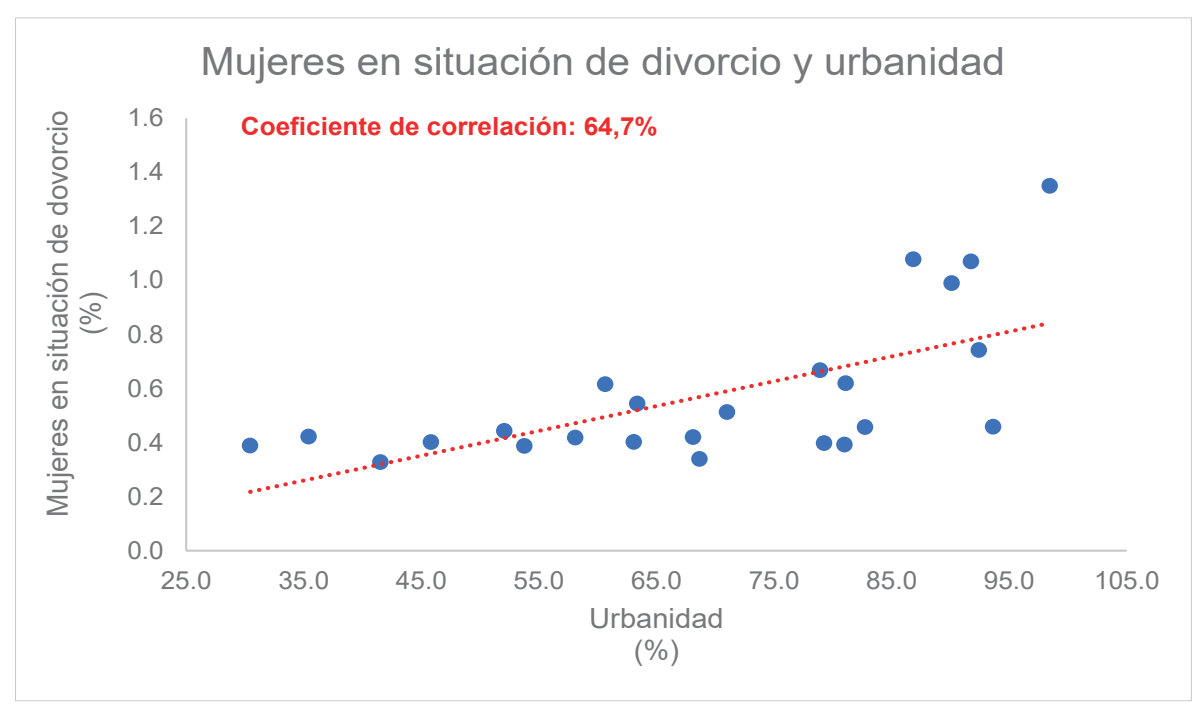

Figura 3. Tasa de divorcio y urbanidad

Fuente: Elaboración propia con base en el Instituto Nacional de Estadística e Informática (2018)

divorcio; es decir, a medida que aumenta la población urbana también la tasa de divorcio tiende a aumentar.

$\mathrm{ED}_{\mathrm{i}}=\mathrm{C}+\alpha \mathrm{JFAP}_{\mathrm{i}}+\beta \mathrm{URB}_{\mathrm{i}}+\gamma \mathrm{FLAP}_{\mathrm{i}}+\mu_{\mathrm{i}}$

Donde:

$\mathrm{ED}_{\mathrm{i}}$ : Mujeres en situación de divorcio del departamento "i"

C:Intercepto

JFAP: Porcentaje de jefas del hogar con alto poder adquisitivo del departamento "i"
URB: Tasa de urbanidad del departamento "i"

FLAP: Porcentaje de la fuerza laboral femenina con educación superior del departamento "i"

$\alpha$ : Coeficiente de la variable JFAP

$\beta$ : Coeficiente de la variable URB

$\gamma$ : Coeficiente de la variable FLAP

$\mu_{\mathrm{it}}$ : Término de error del modelo del departamento "i" 
Como se muestra en la Tabla 5, se encontró una relación positiva entre las variables independientes y las variables dependiente. Es decir:

- A medida que aumenta el porcentaje de jefas de hogar con alto poder adquisitivo, el porcentaje de mujeres en situación de divorcio se eleva.

- A medida que aumenta la tasa de urbanidad, el porcentaje de mujeres en situación de divorcio se eleva.

- A medida que aumenta el porcentaje de la fuerza laboral femenina con educación superior, el porcentaje de mujeres en situación de divorcio se eleva.

$\mathrm{ED}=0.3727+0.0992 \mathrm{JFAP}+0.0005 \mathrm{URB}+0.3515 \mathrm{FLAP}$

Por lo tanto:

- Si el porcentaje de jefas de hogar con alto poder adquisitivo aumenta en un punto porcentual, el porcentaje de mujeres en situación de divorcio se eleva en 0.0992 puntos porcentuales.

- Si la tasa de urbanidad aumenta en un punto porcentual, el porcentaje de mujeres en situación de divorcio se eleva en 0.0005 puntos porcentuales.

\section{DISCUSIÓN}

Los resultados fueron congruentes con el objetivo, ya que se evidenciaron una correlación positiva fuerte de $74.80 \%$ entre la tasa de divorcio y la fuerza laboral femenina con educación superior; entre la tasa de divorcio y el poder adquisitivo de la jefa de hogar presentaron una correlación directa y significativa de $96.8 \%$; y, finalmente, entre la tasa de divorcio y urbanidad presentaron una correlación directa y positiva de $64.70 \%$. Estos resultados coinciden con lo sostenido por el Ministerio de Trabajo y Promoción del Empleo (2017) cuando precisa que "la participación laboral de la mujer, de acuerdo con la literatura económica, ha estado sustentado por el enfoque neoclásico cuyo objetivo es el de maximizar su consumo de bienes y el tiempo de ocio del que dispone" (p. 37). Es así como, bajo este enfoque, "analizar la decisión que toma el individuo de participar o no en el mercado laboral dependerá de que el efecto sustitución (positivo) sea mayor al efecto ingreso (negativo)" (Ministerio de Trabajo y Promoción del Empleo, 2017, p. 1).

Asimismo, los resultados guardan relación con lo precisado por Bremmer y Kesselring (2004), quienes argumentan que las esposas que enfrentan un mayor riesgo de divorcio se protegerán contra ese riesgo con mayores niveles

Tabla 5

Modelo Planteado

Method: Least Squares

Date: $07 / 01 / 19$

Time: 12:08

Included observations: 24

\begin{tabular}{lrrrr}
\multicolumn{1}{c}{ Variable } & Coefficient & Std. Errir & t-Statistic & Prob. \\
\hline C & 0.372686 & 1.116199 & 3.207307 & 0.0044 \\
JFAP & 0.099186 & 0.008262 & 12.00522 & 0.0000 \\
URB & 0.000451 & 0.001224 & 0.368666 & 0.0005 \\
FLAP & 0.351491 & 0.322761 & 1.089079 & 0.0000 \\
R-squared & 0.93521 & Mean dependent var & & 0.570833 \\
Adjusted R-squeared & 0.925491 & S.D. dependent var & 0.277378 \\
S.E. of regression & 0.075714 & Akaike infa criterion & -2.172696 \\
Sum squared resid & 0.114652 & Schwarz criterion & -1.976354 \\
Log likelihood & 30.07235 & Hannan - Quinn crter. & -2.120607 \\
F-statistic & 96.22914 & Durbin - Watson stat & 1.914983 \\
Prob (F-statistic) & 0.000000 & & & \\
\hline
\end{tabular}

Fuente: Elaboración propia con base en el Instituto Nacional de Estadística e Informática (2018) 
de participación en la fuerza laboral. El argumento básico de estos estudios es que la inversión en actividades no relacionadas con el mercado, como la crianza de los hijos, se vuelve relativamente menos productiva (produce un rendimiento esperado más bajo), y la inversión en capital humano, la cual refleja una mejor educación superior por parte de la mujer, hace que se vuelva más productiva (produce un rendimiento esperado más alto) a medida que aumenta la probabilidad de divorcio. Un aumento de la participación femenina en la fuerza de trabajo conduce a un aumento de los conflictos familiares y, en consecuencia, un aumento en la tasa de divorcio. De igual manera, las mujeres con educación superior son más empleables en el mercado laboral, por lo que prefieren realizar un trabajo fuera del hogar. Dependiendo de cualquier causa que origine una crisis dentro del matrimonio, será más fácil tomar la decisión de divorciarse para las mujeres que cuentan con un buen empleo. Como señala Dobson (2017), las mujeres trabajadoras tienen más probabilidades de divorciarse que sus contrapartes que se quedan en casa, haciendo que una mujer sea económicamente independiente casada o divorciada.

Finalmente, Knapton (2018) sostiene que el número de mujeres con poder adquisitivo alto guarda una relación con el incremento de divorcios y se espera que la tendencia continúe cada año; debido a que las mujeres que se valen por sí mismas o han heredado riquezas tienen menos probabilidades de soportar traiciones, maltratos físicos y/o psicológicos por parte de su cónyuge. Si bien algunos pueden criticarlas, muchas de estas mujeres son empresarias o emprendedoras que están en el corazón de la economía y su éxito genera más empleos y más riqueza para el país. Lo anteriormente señalado coincide con los expresado por Mark (2018), quien sostiene que la creciente proporción de los ingresos de las mujeres respecto a los de los hombres, también se ha identificado como un factor que contribuye a la disminución de las tasas de matrimonio. Sin embargo, como manifiesta Carrasco (2017), las explicaciones teóricas para esta conexión asumen una persistencia en los roles de género tradicionales en el matrimonio.

Como agenda futura de investigación, se recomienda realizar más estudios sobre la economía de género, e indagar lo siguiente: ¿Cómo la presencia de la mujer en diversos sectores económicos puede contribuir en la toma de decisiones dentro del seno familiar?

\section{REFERENCIAS}

Ailes, E. (03 de Diciembre de 2020). La pandemia de coronavirus acabó con nuestro matrimonio. BBC News.
Obtenido de: https://www.bbc.com/mundo/noticias-55176072

Benítez,M.E.(2017).Lafamilia:Desdelotradicionalalodiscutible. Revista Novedades en Población, 13(26), 59-68. Obtenido de: http://scielo.sld.cu/scielo.php?script=sci_arttext\&pid=S1817-40782017000200005

Bremmer, D., \& Kesselring, R. (2004). Divorce and Female Labor Force Participation: Evidence from Times-Series Data, Causality Tests, and Cointegration. Atlantic Economic Journal, 32(3), 175-190. doi: 10.1007/ BF02299436

Carrasco, V. (2017). El rol de la mujer peruana en el mercado laboral. Info Capital Humano. Recuperado de: http://www.infocapitalhumano.pe/recursos-humanos/informes/el-rol-de-la-mujer-peruana-en-elmercado-laboral/

De la Piedra, A. (6 de marzo de 2017). Día de la mujer: Solo $24 \%$ de puestos de alta dirección está ocupado por mujeres. Gestión. Recuperado de: https:/gestion.pe/ tendencias/dia-mujer-24-puestos-alta-direccion-ocupado-mujeres-228745

Dobson, R. (10 de julio de 2017). Working women more likely to seek divorce. Independent. Recuperado de: https://www.independent.co.uk/news/uk/ this-britain/working-women-more-likely-to-seek-divorce-5346460.html

Guzmán, J., Reyes, J., Castillo, D., \& Cantos, M. (2020). Factores que inciden en el emprendimiento sostenible de las mujeres ecuatorianas. Revista de Producción Ciencias e Investigación, 4(37), 97-110. https://doi. org/10.29018/issn.2588-1000vol4iss37.2020pp97-110

Instituto Nacional de Estadística e Informática. (2018). Perú. Resultados definitivos. Población Económicamente Activa. Recuperado del sitio de Internet del Instituto Nacional de Estadística e Informática: https:// www.inei.gob.pe/media/MenuRecursivo/publicaciones_digitales/Est/Lib1600/

Knapton, S. (19 de mayo de 2014). Divorce helps fuel rise in female multi-millionaires. The Telegraph. Recuperado de: https://www.telegraph.co.uk/news/10839196/Divorcehelps-fuel-rise-in-female-multi-millionaires.html

Mark, I. (1 de junio de 2000). Divorce Rates, Marriage Rates, and the Problematic Persistence of Traditional Marital Roles. Recuperado de https://papers.ssrn.com/ sol3/papers.cfm?abstract_id=224700 
Moine, M. (2013). Factores que inciden en el acceso de las mujeres a cargos de responsabilidad en la estructura organizacional. Ciencias Económicas, 1(10), 21-45.

Ministerio de Trabajo y Promoción del Empleo. (2017). Informe annual 2016. La mujer en el mercado laboral peruano. Recuperado del sitio de Internet del Ministerio de Trabajo y Promoción del Empleo: https://cdn. www.gob.pe/uploads/document/file/31135/informe_ anual_mujer_mercado_laboral_2016.pdf

Suárez, G. (Junio de 2017). Impacto de los factores socioeconómicos en las relaciones matrimoniales en el Ecuador (Disertación previa a la obtención del título de Economista). Pontificia Universidad Católica del Ecuador, Quito. Obtenido del Repositorio de Tesis de Grado y Posgrado: http://repositorio.puce.edu.ec/handle/22000/6897
Tamez, B. M., \& Ribeiro, M. (2016). El divorcio, indicador de transformación social y familiar con impacto diferencial entre los sexos:estudio realizado en Nuevo León. Papeles de población, 22(90). https://doi.org/10. 22185/24487147.2016.90.040

Uchida, E., Araki, S., \& Murata, K. (25 de Marzo de 2019). Socioeconomic factors affecting marriage, divorce and birth rates in a japanese population. Journal of Biosocial Science, 25(4), 499-507. Obtenido de https://bdsp-ehesp.inist.fr/vibad/index.php?action=getRecordDetail\&lang $=$ fr\&idt $=112387$ 